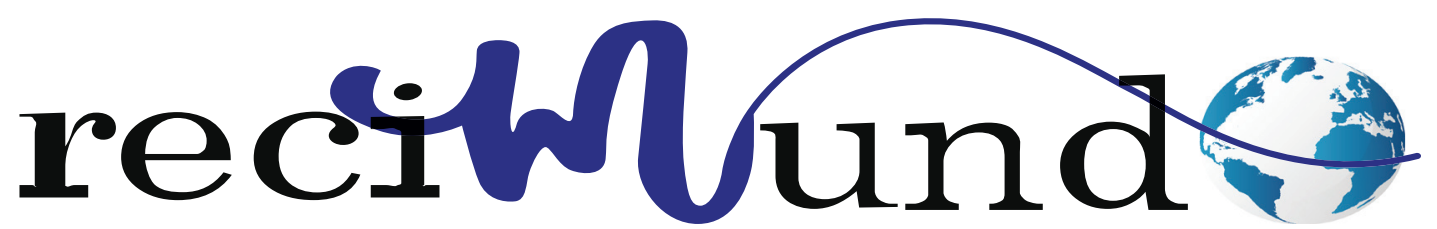

Revista Científica Mundo de la Investigación y el Conocimiento

DOl: 10.26820/recimundo/5.(2).abril.2021.62-70

URL: https://recimundo.com/index.php/es/article/view/1040

EDITORIAL: Saberes del Conocimiento

REVISTA: RECIMUNDO

ISSN: 2588-073X

TIPO DE INVESTIGACIÓN: Artículo de revisión

Código UNESCO: 32 Ciencias Médicas

PAGINAS: $62-70$

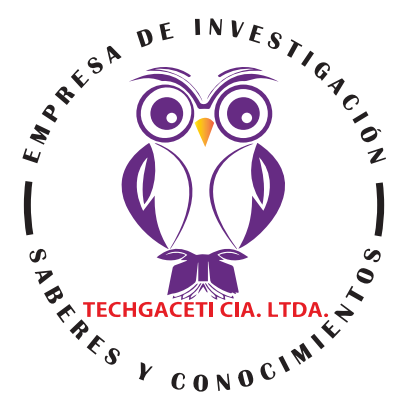

\title{
Terapia con cánula nasal de alto flujo y ventilación mecánica no invasiva
}

High-flow nasal cannula therapy and noninvasive mechanical ventilation

Terapia de cânula nasal de alto fluxo e ventilação mecânica não-invasiva

Martha Alexandra García Montiel'; Javier Enrique Ponce Rodríguez²; Juanissi Lila Turaren González;

Dalma Jannel Morán Santana ${ }^{4}$

RECIBIDO: 15/01/2021 ACEPTADO: 20/03/2021 PUBLICADO: 01/04/2021

1. Médico de la Universidad de Guayaquil; Investigador Independiente; Guayaquil, Ecuador; maralex22@hotmail.com; https://orcid.org/0000-0002-9821-8635

2. Médico de la Universidad de Guayaquil; Investigador Independiente; Guayaquil, Ecuador; javo89_18@hotmail.com; https://orcid.org/0000-0002-8040-6056

3. Médico Cirujano de la Universidad de Oriente; Investigador Independiente; Guayaquil, Ecuador; juanissiturareng@gmail. com; (ID https://orcid.org/0000-0003-2826-6989

4. Médico de la Universidad de Guayaquil; Investigador Independiente; Guayaquil, Ecuador; dalma_1993_@hotmail.com; https://orcid.org/0000-0001-7057-7399

CORRESPONDENCIA

Martha Alexandra García Montiel

maralex22@hotmail.com

Guayaquil, Ecuador

() RECIMUNDO; Editorial Saberes del Conocimiento, 2021 


\title{
RESUMEN
}

La oxigenoterapia con cánula nasal de alto flujo (HFNC) tiene varias ventajas fisiológicas sobre los dispositivos de oxigenoterapia tradicionales, que incluyen una disminución de la resistencia nasofaríngea, lavado del espacio muerto nasofaríngeo, generación de presión positiva en la faringe, aumento del reclutamiento alveolar en los pulmones, las vías respiratorias, mayor fracción de oxígeno inspirado y mejor aclaramiento mucociliar. Recientemente, el uso de HFNC en el tratamiento de pacientes adultos con enfermedades críticas ha aumentado significativamente y ahora se usa en muchos pacientes con una variedad de enfermedades diferentes. Sin embargo, no existen pautas establecidas para orientar el uso seguro y eficaz de la HFNC para estos pacientes. Este artículo de revisión resume la literatura publicada disponible sobre los efectos fisiológicos positivos, mecanismos de acción, y las aplicaciones clínicas de la HFNC, en comparación con los dispositivos de oxigenoterapia tradicionales. La literatura disponible sugiere que la oxigenoterapia HFNC es una modalidad eficaz para el tratamiento temprano de pacientes críticamente adultos.

Palabras clave: Oxigenoterapia, cánula nasal, presión positiva en las vías respiratorias, insuficiencia respiratoria.

\begin{abstract}
High-flow nasal cannula (HFNC) oxygen therapy has several physiological advantages over traditional oxygen therapy devices, including decreased nasopharyngeal resistance, washing out of the nasopharyngeal dead space, generation of positive pressure in the pharynx, increasing alveolar recruitment in the lungs, humidification of the airways, increased fraction of inspired oxygen and improved mucociliary clearance. Recently, the use of HFNC in treating adult critical illness patients has significantly increased, and it is now being used in many patients with a range of different disease conditions. However, there are no established guidelines to direct the safe and effective use of HFNC for these patients. This review article summarizes the available published literature on the positive physiological effects, mechanisms of action, and the clinical applications of HFNC, compared with traditional oxygen therapy devices. The available literature suggests that HFNC oxygen therapy is an effective modality for the early treatment of critically adult patients.
\end{abstract}

Keywords: Oxygen therapy, nasal cannula, positive airway pressure, respiratory failure

\section{RESUMO}

A oxigenoterapia com cânula nasal de alto fluxo (HFNC) tem várias vantagens fisiológicas sobre os dispositivos tradicionais de oxigenoterapia, incluindo diminuição da resistência nasofaríngea, lavagem do espaço morto nasofaríngeo, geração de pressão positiva na faringe, aumento do recrutamento alveolar nos pulmões, umidificação das vias aéreas, aumento da fração inspirada de oxigênio e melhoria da folga mucociliar. Recentemente, o uso de HFNC no tratamento de pacientes adultos com doenças críticas aumentou significativamente, e agora está sendo usado em muitos pacientes com uma gama de diferentes condições de doença. Entretanto, não há diretrizes estabelecidas para direcionar o uso seguro e eficaz de HFNC para esses pacientes. Este artigo de revisão resume a literatura publicada disponível sobre os efeitos fisiológicos positivos, mecanismos de ação e as aplicações clínicas de HFNC, em comparação com os dispositivos tradicionais de oxigenoterapia. A literatura disponível sugere que a oxigenoterapia com HFNC é uma modalidade eficaz para o tratamento precoce de pacientes criticamente adultos.

Palavras-chave: Oxigenoterapia, cânula nasal, pressão positiva nas vias aéreas, insuficiência respiratória. 


\section{Introducción}

La cánula nasal se ha convertido en el dispositivo de elección en la medicina moderna para el suministro de oxígeno suplementario a pacientes con enfermedades hipoxémicas no hipercarbicas. "Dichos pacientes pueden requerir terapia con oxígeno inspiratorio alto, típicamente en el rango de 30 a 120 I / min" (Ward, 2013). Sin embargo, los dispositivos tradicionales de suministro de oxígeno, como la cánula nasal de bajo flujo, las máscaras sin reservorio y con bolsa de reservorio, sistemas de aerosoles de gran volumen (utilizados en una fracción de oxígeno inspirado [FiO2]> 0,4), solo puede suministrar una velocidad de flujo de oxígeno de 6 a 15 I/ min, lo que es insuficiente para muchos pacientes.

De acuerdo con Roca, Riera, \& Torres, 2010 "la cánula nasal de alto flujo (HFNC) es un novedoso dispositivo de suministro de oxígeno capaz de suministrar hasta un 100\% de oxígeno humedecido y calentado a un caudal máximo de 60 I / min". Se ha demostrado que confiere muchas ventajas potenciales en comparación con los dispositivos tradicionales de suministro de oxígeno y conduce a mejores resultados fisiológicos.

En los últimos años, la HFNC se está utilizando cada vez más con pacientes adultos con enfermedades críticas, donde se ha aplicado con éxito a una cantidad de pacientes con una variedad de enfermedades diferentes. Sin embargo, "otra investigación señaló que el tratamiento con HFNC no hizo ninguna diferencia en el resultado en comparación con la oxigenoterapia estándar o la ventilación no invasiva" (Frat, Thille, \& Mercat, 2015).

Esta revisión tiene como objetivo resumir y discutir la comprensión actual con respecto a los efectos fisiológicos de la HFNC, sus mecanismos de acción y su aplicación en una variedad de entornos clínicos. Esta información ayudará a los médicos a deter- minar los posibles beneficios y limitaciones en el uso clínico que afectan los resultados de la oxigenoterapia HFNC e informará las decisiones de estrategia clínica relacionadas con los pacientes adultos que requieren oxígeno suplementario.

\section{Metodología}

Para el desarrollo de este proceso investigativo, se plantea como metodología la encaminada hacia una orientación científica particular que se encuentra determinada por la necesidad de indagar en forma precisa y coherente una situación, en tal sentido Davila, (2015) define la metodología "como aquellos pasos anteriores que son seleccionados por el investigador para lograr resultados favorables que le ayuden a plantear nuevas ideas" (p.66)

Lo citado por el autor, lleva a entender que el desarrollo de la acción investigativa busca simplemente coordinar acciones enmarcadas en una revisión bibliográfica con el fin de complementar ideas previas relacionadas Terapia con cánula nasal de alto flujo y ventilación mecánica no invasiva a través de una revisión de literatura, para así finalmente elaborar un cuerpo de consideraciones generales que ayuden a ampliar el interés propuesto.

\section{Tipo de Investigación}

Dentro de toda práctica investigativa, se precisan acciones de carácter metodológico mediante las cuales se logra conocer y proyectar los eventos posibles que la determinan. En este sentido, la presente investigación corresponde al tipo documental, definido por Castro (2016), "se ocupa del estudio de problemas planteados a nivel teórico, la información requerida para abordarlos se encuentra básicamente en materiales impresos, audiovisuales y / o electrónicos". (p.41). 
En consideración a esta definición, la orientación metodológica incluye la oportunidad de cumplir con una serie de actividades inherentes a la revisión y lectura de diversos documentos, donde se encuentran ideas explicitas relacionadas con los tópicos encargados de identificar una característica inmersa en el estudio. Por lo tanto, se realizaron continuas interpretaciones con el claro propósito de revisar aquellas apreciaciones propuestas por diferentes investigadores en relación al tema de interés, para luego dar la respectiva argumentación a los planteamientos, en función a las necesidades encontradas en la investigación, apoyados en las herramientas tecnológicas para la búsqueda de trabajos con valor científico disponibles en la web que tenían conexión con el objetivo principal de la investigación.

\section{Fuentes Documentales}

El análisis correspondiente a las características que predomina en el tema seleccionado, llevan a incluir diferentes fuentes documentales encargadas de darle el respectivo valor científico y en ese sentido cumplir con la valoración de los hechos a fin de generar nuevos criterios que sirven de referencia a otros procesos investigativos. Para Castro,(2016) las fuentes documentales incorporadas en la investigación documental o bibliográfica, "representa la suma de materiales sistemáticos que son revisados en forma rigurosa y profunda para llegar a un análisis del fenómeno" (p.41). Por lo tanto, se procedió a cumplir con la lectura previa determinada para encontrar aquellos aspectos estrechamente vinculados con el tema, con el fin de explicar mediante un desarrollo las respectivas apreciaciones generales de importancia.

\section{Técnicas para la Recolección de la Infor- mación}

La conducción de la investigación para ser realizada en función a las particularidades que determinan a los estudios documenta- les, tiene como fin el desarrollo de un conjunto de acciones encargadas de llevar a la selección de técnicas estrechamente vinculadas con las características del estudio. Bolívar, (2015), refiere, que es "una técnica particular para aportar ayuda a los procedimientos de selección de las ideas primarias y secundarias". (p.71).

Tal como lo expresa, Bolívar, (2015) "Las técnicas documentales proporcionan las herramientas esenciales y determinantes para responder a los objetivos formulados y llegar a resultados efectivos" (p. 58). Es decir, para responder con eficiencia a las necesidades investigativas, se introdujeron como técnica de recolección el método inductivo, que hizo posible llevar a cabo una valoración de los hechos de forma particular para llegar a la explicación desde una visión general. El autor Bolívar, (2015) tambien expresa que las técnicas de procesamiento de datos en los estudios documentales "son las encargadas de ofrecer al investigador la visión o pasos que deben cumplir durante su ejercicio, cada una de ellas debe estar en correspondencia con el nivel a emplear" (p. 123). Esto indica, que para llevar a cabo el procesamiento de los datos obtenidos una vez aplicadas las técnicas seleccionadas, tales como: fichas de resumen, textual, registros descriptivos entre otros, los mismos se deben ajustar al nivel que ha sido seleccionado.

\section{Resultados}

\section{Mecanismos potenciales de beneficio clí- nico}

Se ha demostrado que la terapia con cánula nasal de alto flujo produce una variedad de efectos fisiológicos beneficiosos.

Los estudios han demostrado que su uso mejora la oxigenación, genera presión positiva en las vías respiratorias, mejora el volumen pulmonar al final de la inspiración, debilita la resistencia inspiratoria, reduce el

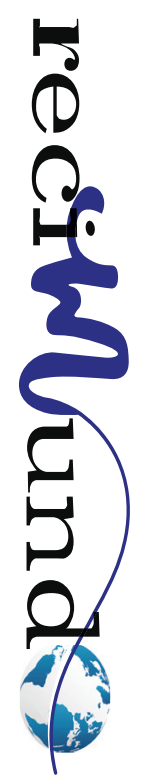


trabajo metabólico asociado con el acondicionamiento de gases, elimina el espacio muerto nasofaríngeo y aumenta la capacidad residual funcional (Dysart, Miller, \& Wolfson, 2009).

Los efectos fisiológicos sugieren que el tratamiento con HFNC podría ser eficaz en el tratamiento de la depresión respiratoria resultante de muchas causas diferentes. Además, la evidencia sugiere que los pacientes toleran mejor la HFNC y es más cómoda que otras aplicaciones de oxigenoterapia tradicionales.

\section{Mecanismos de acción de la terapia de HFNC}

\section{Lavado del espacio muerto faríngeo}

Un mecanismo de acción de la terapia con HFNC podría ser a través de su efecto en el lavado del espacio muerto de la cavidad faríngea, reduciendo así el espacio muerto general y conduciendo a una mejora en la ventilación alveolar en los pulmones. Sin embargo, debido a las complejidades de la disección de la vía aérea faríngea y la incapacidad de medir el flujo de gas, es difícil estudiar los mecanismos precisos del lavado del espacio muerto. Aunque los flujos más altos pueden reducir el espacio muerto, la relación aún no se ha determinado. Por lo tanto, se sugiere que la terapia con HFNC no se use para cuidar a pacientes con niveles elevados de $\mathrm{CO}^{2}$.

En 2004, se realizó un estudio prospectivo que tenía como objetivo comparar los efectos de la HFNC con los de los dispositivos tradicionales de inhalación de oxígeno de bajo flujo para pacientes con enfermedad pulmonar obstructiva crónica (EPOC). "Los autores encontraron que los pacientes que usaban dispositivos HFNC podían hacer ejercicio durante un período de tiempo más largo que aquellos que usandispositivos de suministro de oxígeno de bajo flujo(10,0 $\pm 2,4$ minversus $8,2 \pm 4,3$ min, res- pectivamente)" (Chatila, Nugent, \& Vance, 2004). Estos pacientes también experimentaron menos disnea, mejor funcionamiento respiratorio y tensión arterial más baja. Los autores concluyeron que el uso de la terapia HFNC mejora el rendimiento del ejercicio en pacientes con EPOC, en parte al mejorar la oxigenación.

Más recientemente, se llevó a cabo un estudio para confirmar el efecto de lavado del espacio muerto de la HFNC y su influencia en la eliminación y oxigenación de CO2. "Trece lechones recién nacidos con lesiones pulmonares agudas recibieron apoyo con HFNC con un flujo de oxígeno que variaba de 2 a 8 I. / min" (Frizzola, Miller, \& Rodriguez, 2011). Se utilizaron cánulas de una y dos puntas para lograr fugas de nivel alto y bajo alrededor de las puntas nasales. Los análisis de hemodinámica, respiración y gases en sangre se realizaron en cada entorno experimental 10 minutos después de que se alcanzó el equilibrio fisiológico.

Los autores concluyeron que la HFNC promovió el intercambio gaseoso regulando el flujo de oxígeno, que la administración a través de la cánula de doble punta logró un mayor efecto sobre la oxigenación y que la administración a través de la cánula de una sola punta tuvo un mayor impacto en la eliminación de CO2 (Frizzola, Miller, \& Rodriguez, 2011).

\section{Reducción de la resistencia nasofaríngea}

Otro efecto importante observado de la terapia con HFNC es la reducción de la resistencia al flujo de aire nasofaríngeo. "Después de analizar los bucles de volumen de flujo en la nasofaringe, la investigación mostró que la nasofaringe tiene una capacidad de expansión que da como resultado una resistencia alterable" (Arora, Mahajan, \& Zidan, 2012).

Un estudio que comparó el efecto de la tasa de flujo sobre la resistencia en presión 
positiva continua en las vías respiratorias (CPAP), cánula nasal y HFNC, encontraron que el orden de resistencia de estos diferentes métodos de suministro de oxígeno era el siguiente: HFNC $783 \mathrm{cmH} 2 \mathrm{O} / \mathrm{I} /$ s> CPAP $280 \mathrm{cmH} 2 \mathrm{O} / \mathrm{l} / \mathrm{s}$. Sin embargo, el mecanismo más probable por el cual la HFNC reduce la resistencia de la nasofaringe inspiratoria es probable ser aumentando el flujo inspiratorio (Arora, Mahajan, \& Zidan, 2012).

\section{Presión espiratoria positiva}

La cánula nasal de alto flujo genera presión faríngea positiva, que produce una cierta cantidad de presión de expansión pulmonar y promueve el reclutamiento alveolar.

En un estudio reciente en el que se registró la presión faríngea con una tasa de flujo de oxígeno HFNC de 0 a 601 / min, se encontró que la presión espiratoria con la boca cerrada era significativamente más alta que con la boca abierta $(P<0,001)$. Este estudio demostró que existe un cierto grado de CPAP producido por la terapia HFNC, que es tanto dependiente del flujo y dependiente de la posición de la boca (abierta versus cerrada ) (Groves \& Tobin, 2017).

Este efecto se confirmó al estudiar la correlación entre la tasa de flujo y la presión en el contexto del sistema de oxigenoterapia de flujo alto nasal Opti flow TM22. Las mediciones se realizaron con oxígeno de flujo alto nasal a caudales de 30, 40 y 50 I / min,con la boca del paciente tanto abierta como cerrada. Las presiones se registraron durante 1 minuto de respiración y los flujos promedio se calcularon mediante un promedio simple. "Los autores encontraron que, durante la terapia de oxígeno de flujo alto nasal, la presión nasofaríngea media aumentaba a medida que la tasa de flujo de oxígeno aumentaba aumentado" (Parke, Eccleston, \& McGuinness, 2011).

\section{Reclutamiento alveolar}

El dispositivo HFNC puede reducir la hipoxemia a través de varios mecanismos, aliviando así los síndromes de depresión respiratoria. Al generar presión positiva en las vías respiratorias, la HFNC genera una presión de expansión pulmonar, lo que promueve el reclutamiento alveolar. Este efecto se ha demostrado en voluntarios sanos y en pacientes que se recuperan de una cirugía cardíaca, pero aún se desconocen los efectos de la HFNC sobre el volumen pulmonar. Un estudio evaluó a veinte pacientes que se recuperaban de una cirugía cardíaca y recibían oxigenoterapia con HFNC. Los autores Corley, Caruana, \& Barnett, (2011) utilizaron Tomografía de impedancia eléctrica para evaluar el impacto de la HFNC en la presión de las vías respiratorias, el volumen pulmonar al final de la espiración (EELV) y la relación entre ambos. Los autores concluyeron que la oxigenoterapia por HFNC redujo la frecuencia respiratoria y mejoró la oxigenación mediante el aumento tanto del EELV como del volumen corriente, y sugirió que sería más beneficioso en pacientes con índices de masa corporal más altos (p. 998).

\section{Humidificación y tolerancia}

La necesidad de calentar y humidificar el dispositivo HFNC durante su uso ha sido un punto de controversia dentro de la literatura publicada. En "comparación con otros dispositivos de oxigenoterapia, varios estudios han informado que el uso de la terapia HFNC da como resultado una frecuencia respiratoria más baja, y que el dispositivo es mejor tolerado por los pacientes, además de ser más cómodo" (Groves \& Tobin, 2017). Al emplear un humidificador calentado junto con la terapia de HFNC, se observó una reducción de los síntomas de sequedad, que fue regulada por aumento del nivel de humedad.

Otro estudio encontró que "la comodidad de los pacientes fue mayor durante las se-

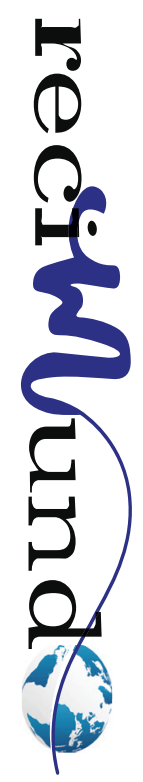


siones de HFNC en comparación con las sesiones de ventilación no invasiva en la insuficiencia respiratoria hipoxémica aguda" (Frat, Brugiere, \& Ragot, 2015). Debido a que el oxígeno frío y seco generado por el dispositivo de HFNC aumenta la resistencia al flujo de aire, es necesario calentar y humedecer el aire. Este sistema de calor y humidificación también puede influir indirectamente en la oxigenación.

\section{Fracción de oxígeno inspirado y aclara- miento mucociliar}

Otros mecanismos por los cuales la terapia con HFNC puede producir efectos positivos incluyen la capacidad de regular con precisión la $\mathrm{FiO} 2$ de un paciente y lograr una mejor limpieza mucociliar. Los sistemas de defensa primaria de las vías respiratorias incluyen estornudos, náuseas, tos y filtración natural. El sistema de defensa secundario, el sistema de transporte mucociliar, atrapa y neutraliza los contaminantes inhalados y luego los transporta fuera del tracto respiratorio. Sin embargo, el sistema de transporte mucociliar es extremadamente sensible a la humedad. Al administrar un flujo humidificado de oxígeno, "la HFNC puede ayudar a los pacientes a mantener el funcionamiento de su sistema de defensa de las vías respiratorias secundarias para eliminar las excreciones de manera eficiente, reduciendo así el riesgo de infección respiratoria" (Restrepo \& Walsh, 2012).

\section{Aplicación de la HFNC en varios entornos clínicos}

Como se mencionó anteriormente, la terapia con HFNC puede tener muchos efectos fisiológicos positivos en el sistema de las vías respiratorias. Sus ventajas sobre los dispositivos de oxigenoterapia más tradicionales han llevado a los investigadores a estudiar la terapia HFNC en una variedad de aplicaciones clínicas, entre las que se exponen

\section{Insuficiencia respiratoria hipoxémica aguda}

Entre los pacientes con insuficiencia respiratoria hipoxémica aguda (ICAH), "la terapia con HFNC se correlaciona con una frecuencia respiratoria más baja, y se tolera mejor y es más cómoda que las alternativas más tradicionales" (Arora, Mahajan, \& Zidan, 2012). Se realizó un estudio para comparar qué tan cómoda se percibía la oxigenoterapia administrada a través de la HFNC compararse con la oxigenoterapia a través de una mascarilla facial tradicional en 20 pacientes con insuficiencia respiratoria aguda. En tal sentido, "se informó que la HFNC se asoció con menos disnea y sequedad bucal, y fue más cómoda. HFNC también se asoció con una mayor presión parcial de oxígeno arterial ( $\mathrm{PaO} 2$ ) y frecuencia respiratoria inferior" (Roca, Riera, \& Torres, 2010).

Más recientemente, "un estudio observacional prospectivo investigó el impacto de la HFNC en comparación con la oxigenoterapia tradicional en pacientes con insuficiencia respiratoria aguda, y confirmó los beneficios positivos de la HFNC antes mencionados" (Sztrymf, Messika, \& Mayot, 2012). La terapia con HFNC se asoció nuevamente con una frecuencia respiratoria más baja, un nivel de saturación de oxígeno más alto y una PaO2 más alta que los otros modos de administración probados.

\section{Período posterior a la extubación}

Se sabe que la reintubación de los pacientes se asocia con un aumento de la duración de la estancia en la unidad de cuidados intensivos (UCl) y hospitalaria y con un aumento de la mortalidad. Recientemente, con el mayor uso de dispositivos HFNC y sus efectos beneficiosos comprobados y una mejor tolerancia por parte de los pacientes, se ha ampliado la investigación para investigar su uso para prevenir o tratar la insuficiencia respiratoria posterior a la extubación. 


\section{Período previo a la intubación}

La intubación se realiza a menudo para pacientes hipoxémicos e inestables en la UCl y se asocia con complicaciones graves que ponen en peligro la vida. La VNI se puede aplicar para mejorar la oxigenación antes de que tenga lugar la intubación traqueal. "Debido a los efectos beneficiosos y la mejor tolerancia de la terapia con HFNC, se ha sugerido que podría usarse para administrar oxígeno durante el período apneico de la intubación traqueal en lugar de otros dispositivos de oxígeno tradicionales (mascarillas)" (Ricard, 2012).

En un estudio de investigación experimental en el que participaron ocho lechones anestesiados con lesión pulmonar inducida por lavado pulmonar, se encontró que el uso directo de la terapia con HFNC a una velocidad de flujo de 10 I/ min a través de la faringe durante la intubación alivió notablemente la hipoxemia durante la apnea (Engstrom, Hedenstierna, \& Larsson, 2010).

Esto indicó que la terapia con HFNC podría utilizarse para pacientes de $\mathrm{UCl}$ durante la intubación traqueal.

\section{Infección respiratoria}

Se han realizado investigaciones limitadas sobre el uso de la oxigenoterapia HFNC en el tratamiento de la infección respiratoria aguda grave (IRAG). "En un análisis posthoc de un solo centro, los investigadores evaluaron la efectividad de la oxigenoterapia por HFNC en pacientes de la UCI que ingresaron con IRAG debido al brote de influenza A / H1N1 de 2009" (Rello, Perez, \& Roca, 2012). De los 25 pacientes adultos con IRAG no intubados en este estudio, 20 no pudieron sostener la saturación de oxígeno por encima del 92\% con la oxigenoterapia tradicional y necesitaron terapia HFNC, que fue exitosa en nueve casos (45\%). Con la oxigenoterapia HFNC, los no respondedores presentaron $\mathrm{PaO} 2$ / FiO2 más baja 6 horas después, y requirieron mayores tasas de flujo de oxígeno. No se informaron infecciones secundarias en los trabajadores de la salud. Tampoco hubo neumonías nosocomiales (adquiridas en el hospital) durante la terapia de oxígeno HFNC.

La bronquiolitis es la causa más común de infección del tracto respiratorio inferior. Si bien la evidencia relacionada con la efectividad del tratamiento con HFNC en pacientes con bronquiolitis está disponible para lactantes, es limitada en adultos. Los estudios sobre el uso de la terapia HFNC en bebés con bronquiolitis sugieren que es un modo seguro de asistencia respiratoria que puede proporcionar una alternativa a la CPAP nasal.

\section{Conclusiones}

Durante el desarrollo del proceso investigativo se observó que la oxigenoterapia con cánula nasal de alto flujo es una alternativa de aplicación clínica valiosa a la oxigenoterapia convencional para pacientes críticamente enfermos. Parecería ser eficaz para el tratamiento de pacientes con insuficiencia respiratoria, infección respiratoria y enfermedad obstructiva de las vías respiratorias, ya sea durante el período posterior a la extubación o antes de la intubación.

Sin embargo, la presión positiva al final de la espiración es difícil de medir en estos entornos debido a la naturaleza no invasiva de la terapia de HFNC y se han observado fugas de aire. A pesar de estas incertidumbres, un conjunto de pruebas cada vez mayor indica que la oxigenoterapia HFNC es una modalidad de tratamiento eficaz para el tratamiento temprano de pacientes adultos en estado crítico.

No obstante, se requieren más investigaciones para confirmar los efectos a largo plazo de la HFNC e identificar la población de pacientes adultos para quienes podría ser más beneficioso en aras de brindar un me-

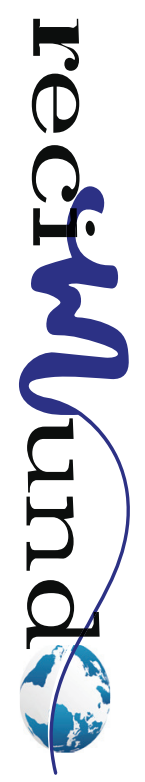


jor uso de este tipo de terapias, que sirva como una alternativa beneficiosa para los pacientes que lo necesiten.

\section{Bibliografía}

Arora, B., Mahajan, P., \& Zidan, M. (2012). Nasopharyngeal airway pressures in bron- chiolitis patients treated with high-flow nasal cannula oxygen therapy. Pediatr Emerg Care, 1179-1184.

Bolívar, J. (2015). Investigación Documental. México. Pax.

Castro, J. (2016). Técnicas Documentales. México. Limusa.

Chatila, W., Nugent, T., \& Vance, G. (2004). The effects of high-flow vs low-flow oxygen on exercise in advanced obstructive airways disease. Chest , $1108-1115$.

Corley, A., Caruana, L., \& Barnett, A. (2011). Oxygen delivery through high-flow nasal cannulae increase end-expiratory lung volume and reduce respiratory rate in post- cardiac surgical patients. $\mathrm{Br} \mathrm{J}$ Anaesth, 998-1004.

Davila, A. (2015). Diccionario de Términos Científicos. . Caracas: Editorial Oasis.

Dysart, K., Miller, T., \& Wolfson, M. (2009). Research in high flow therapy: mechanisms of action. Respir Med, 1400-1405.

Engstrom, J., Hedenstierna, G., \& Larsson, A. (2010). Pharyngeal oxygen administration increases the time to serious desaturation at intubation in acute lung injury: an experimental study. Crit Care, 93.

Frat, J., Brugiere, B., \& Ragot, S. (2015). Sequential application of oxygen therapy via high-flow nasal cannula and noninvasive ventilation in acute res- piratory failure: an observational pilot study. Respir Care, 170-178.

Frat, J., Thille, A., \& Mercat, A. (2015). High- flow oxygen through nasal cannula in acute hypoxemic respiratory failure. N Engl J Med, 2185-2196.

Frizzola, M., Miller, T., \& Rodriguez, M. (2011). High-flow nasal cannula: impact on oxy-genation and ventilation in an acute lung injury model. Pediatr Pulmonol, 67-74.

Groves, N., \& Tobin, A. (2017). High flow nasal oxygen generates positive airway pressure in adult volunteers. Aust Crit Care, 126-131.

Parke, R., Eccleston, M., \& McGuinness, S. (2011). The effects of flow on airway pressure during nasal high-flow oxygen therapy. Respir Care, 1151-1155.

Rello, J., Perez, M., \& Roca, O. (2012). High-flow nasal therapy in adults with severe acute respiratory infection: a cohort study in patients with 2009 influenza A/H1N1v. J Crit Care, 434-439.

Restrepo, R., \& Walsh, B. (2012). American Association for Respiratory Care. Humidification during invasive and nonin- vasive mechanical ventilation: 2012. Respir Care, 782-788.

Ricard, J. (2012). High flow nasal oxygen in acute respiratory failure. Minerva Anestesiol, 836-841.

Roca, O., Riera, J., \& Torres, F. (2010). High-flow oxygen therapy in acute respiratory failure. Respir Care, 408-413.

Sztrymf, B., Messika, J., \& Mayot, T. (2012). Impact of high-flow nasal cannula oxygen therapy on intensive care unit patients with acute respiratory failure: a prospective observa- tional study. J Crit Care, 324.e9-324.e13.

Ward, J. (2013). High-flow oxygen administration by nasal cannula for adult and perinatal patients. Respir Care , 98-122.

\section{CITAR ESTE ARTICULO:}

García Montiel, M. A., Ponce Rodríguez, J. E., Turaren González, J. L., \& Morán Santana, D. J. (2021). Terapia con cánula nasal de alto flujo y ventilación mecánica no invasiva. RECIMUNDO, 5(2), 62-70. https://doi.org/10.26820/ recimundo/5.(2).abril.2021.62-70 